\title{
ESTRATEGIAS DE AFRONTAMIENTO EN EL PERFIL DESCRIPTIVO DEL ESTRÉS ACADÉMICO DE LOS ESTUDIANTES UNIVERSITARIOS
}

\author{
COATING STRATEGIES IN THE DESCRIPTIVE PROFILE OF ACADEMIC STRESS OF \\ UNIVERSITY STUDENTS
}

\section{Referencia del artículo}

González, H. (2019). Estrategias de afrontamiento en el perfil descriptivo del estrés académico de los estudiantes universitarios. Revista Científica del SEP. 2(1), 25-30. DOI: https://doi.org/10.36958/sep.v2i01.14

\author{
Humberto González Castillo \\ gigantepreclaro@hotmail.com \\ Maestría en Docencia Universitaria con Orientación en Estrategias de Aprendizaje \\ Centro Universitario de Oriente \\ Universidad de San Carlos de Guatemala \\ Fecha de recepción: 19/03/2019 \\ Fecha de aceptación: 25/10/2019
}

\section{RESUMEN}

El objetivo principal de este artículo es determinar el nivel de frecuencia con que se presenta el componente "Estrategias de Afrontamiento" en el perfil descriptivo del estrés académico, de alumnos de último año de carreras plan fin de semana CUNORI-USAC. Comparando los resultados, conforme al uso e impacto de este componente, entre las diferentes carreras investigadas.

Por la táctica teórico-metodológica utilizada en el estudio, se le consideró de tipo cuantitativo-cualitativo, con un alcance descriptivo; en el cual se tomó la población totalde estudiantes, 150 participantes respectivamente, que cumplieron con los criterios de selección. El estudio se cimentó en la propuesta teórica del enfoque SistémicoCognoscitivista de Arturo Barraza (2006); y la recolección de datos mediante su Inventario SISCO, en el cual se identifican tres componentes del estrés académico: estresores, síntomas y estrategias de afrontamiento, estas últimas son las que se toman para direccionar este artículo. Los resultados indican que el $47.3 \%$ de estudiantes, conforme análisis de frecuencias y porcentajes, utilizan eventualmente estrategias de afrontamiento. En contraparte la población a estudio presentó un nivel de estrés académico que, al centrarse enla relación entre los ítems y el puntaje global del inventario, permiten afirmar que el estrés académico, presente en los sujetos de investigación, se encuentra en un nivel "severo", con un $82.7 \%$ según rangos y criterios establecidos, con una intensidad mediamente alta.

Por lo que se considera que el plan de estudios, de carreras fin de semana aporta elevados niveles de estrés académicoa los estudiantes. Se evidencia que las estrategias de afrontamiento no fueron efectivas, debido a que el empleo de las mismas no redujo el nivel de estrés autopercibido.

\section{PALABRAS CLAVES}

Estresores, síntomas, estrategias de afrontamiento, estrés académico

\begin{abstract}
The main objective of this article is to determine the level of frequency with which the component "Coping Strategies" is presented in the descriptive profile of the academic stress of seniors of the CUNORI-USAC weekend plan races. Comparing the results, according to the use and impact of this component, between the different careers investigated.
\end{abstract}

For the theoretical methodological tactics used in the study, it was considered quantitative-qualitative, with a descriptive scope; in which the total population of students was taken; 150 participants respectively, who met the selection criteria. The study was based on the theoretical proposal of the Systemic-Cognoscitivist approach of Arturo Barraza (2006); and the collection of data through its SISCO Inventory, in which three components of academic stress are identified: stressors, symptoms and coping strategies, the latter being what is taken to address this article. The results indicate that 47.3\% of students, according to analysis of frequencies and percentages, eventually use coping strategies. In contrast in study population presented a level of academic stress that by focusing on the relationship between the items and the overall score of the inventory, allow to affirm that academic stress, present in research subjects, is at a "severe" level, with $82.7 \%$ according to established ranges and criteria, with a medium-high intensity.

As it is considered that the curriculum, weekend careers bring high levels of academic stress to the students. It is evident that coping strategies were not effective, because the use of them did not reduce the level of self-perceived stress.

\section{KEYWORDS}

Stressors, symptoms, coping strategies, academic stress 


\section{INTRODUCCIÓN}

Definir el estrés es una tarea compleja debido a que las percepciones individuales del mismo difieren considerablemente. La definición que goza de mayor aceptación, es la propuesta desde el punto de vista de la psicología "es la respuesta del sistema nervioso a un acontecimiento o una situación que se percibe como una amenaza. Esta respuesta se conoce informalmente como mecanismo de lucha o huida, ya que las hormonas (como la adrenalina) que el organismo secreta al sentir miedo proporcionan al cuerpo la potencia física para enfrentarse al peligro o bien huir" (Labrador, Crespo, Cruzado, \& Vallejo, 1995).

Desde que el estudiante cumple con el perfil de ingreso y es aprobado para formar parte del estudiantado de un centro universitario, su vida toma otro giro y su rutina diaria se rompe para pasar a otro orden diferente, donde se dan cambios que pueden generar en menor o mayor grado, estrés. Es posible que esta persona no cuente con el debido conocimiento de sí mismo y por lo tanto no sepa cómo afrontar esas situaciones, generándole enfermedades que pueden afectar su salud física y mental.

La tensión originada por el estrés, según Ticona et al. (2010), es necesaria para el desarrollo de la personalidad del estudiante; no obstante, cuando el estrés sobrepasó los niveles óptimos, ocasiona el agotamiento de energías, deteriora el desempeño y seguramente puede llegar a dañar la salud. Algunos estudiantes, ante estas situaciones ponen en marcha una serie de estrategias de afrontamiento y tratan de superar las exigencias demandadas, buscando alivio a esa tensión; sin embargo, existe una gran parte de universitarios que carecen de esas estrategias o toman las inadecuadas. De allí la importancia de la capacidad de afrontamiento ante el estrés académico, evitar o disminuir la vulnerabilidad ante el mismo.

El concepto de afrontamiento fue formulado por Lazarus \& Folkman (1986), quienes indican que, el afrontamiento tendrá un proceso variable; las estrategias de afrontamiento no siempre serán las mismas, todo dependerá de la situación; algunas veces el individuo precisará contar con estrategias que le sirvan de defensa y en otras ocasiones necesitará estrategias que le sirvan para resolver el problema. De acuerdo a los autores, estas respuestas de afrontamiento se dividen en dos categorías diferentes: a) dirigidas al problema y b) dirigidas a la emoción. Las continuas quejas y los cambios desafiantes del estudiante universitario; especialmente los del último año de estudios; vienen a influir directamente en el desempeño de las habilidades de afrontamiento cognitivas, afectivas, operativas y sociales; que por lo general, son los de enfrentar elementos de tensión física, psicológica, y de comportamiento.

Con base en la investigación realizada con alumnos de último año de carreras plan fin de semana del Centro Universitario de Oriente, siendo éstas: Licenciatura en Pedagogía y Administración Educativa, Licenciatura en Administración de Empresas, Licenciatura en Ciencias de la Comunicación y Licenciatura en Contaduría Pública y Auditoría; se analizó el nivel de estrés académico presente en los estudiante de último año de carreras referidas, para determinar cómo es afectado por la actividad universitaria y donde uno de sus objetivos principales fue determinar el nivel de frecuencia con que se presenta el componente "Estrategias de Afrontamiento" (con habilidad asertiva, elaborando un plan y ejecución de mis tareas, tratándome elogiosamente, refugio en la religiosidad, buscando información sobre la situación, ventilando y haciendo confidencias) componente tridimensional del Modelo Sistémico Cognoscitivista del estrés académico, mismo en el cual gira este artículo.

Lo anterior lleva a adelantar una concreción tendiente a explicar la situación planteada, en el sentido de que las manifestaciones de estrés autopercibidas por estudiantes antes mencionados, son debido a estresores mismos y a la debilidad del uso de estrategias de afrontamiento.

\section{MATERIALES Y MÉTODOS}

En lo que respecta al Inventario SISCO, cuenta con los siguientes apartados: Sección I, contiene las características sociodemográficas y situacionales: Tipo de carrera que cursa, género, edad, estado civil, número de hijos, entre otros. Sección II: contiene los componentes o dimensiones del estrés académico. Serie I: contiene al ítem 1: pregunta directa para incluir o no en la investigación al sujeto de estudio. Serie II: contiene cuadro con escala del 1 al 5 para señalar el nivel de preocupación o nerviosismo del estudiante.

La serie III: contiene los ítems de las preguntas 3, 4 y 5: 29 ítems que determinan la frecuencia en que afecta el estrés académico; divididos de la siguiente manera: 8 ítems para determinar la frecuencia de los estresores presentes en los estudiantes, 15 ítems que determinan los síntomas físicos, psicológicos y del comportamiento social y 6 ítems para determinar la frecuencia que utilizan diferentes estrategias de afrontamiento al estrés académico. El valor mínimo que un estudiante pudo obtener en su calificación es $0 \%$ y el máximo es $100 \%$. 
La guía de entrevista estuvo conformada por 13 preguntas relacionadas al objeto de estudio y sus variables, incluyó preguntas cerradas con un apartado para que el entrevistado indicara la razón de su respuesta. La guía de observación en su estructura conlleva 15 enunciados de observación sobre el estrés académico, permitiendo que la información pudiera estandarizarse y ser más objetiva, excluyendo los juicios subjetivos de los informantes y del investigador. Además, se obtuvieron directamente los datos de la realidad empírica, sin intermediarios ni distorsiones de la información.

La investigación se suministró con un enfoque mixto, partiendo de que las variables fueran analizadas cuantitativa y cualitativamente. Permitiendo describir, generalidades del objeto de estudio y las variables moduladoras. El método hipotético-deductivo, permitió poner a prueba algunas hipótesis, que buscaban básicamente comprobar el nivel de frecuencia de estrés académico en los estudiantes investigados.

Al usar el método deductivo se estuvo guiando el proceso de manera lógica, con base en las deducciones que se pueda desarrollar, teniendo como punto de partida o referencia, la diagramación referenciada y enmarcada siempre dentro de los parámetros de acción del método científico y del modelo teórico seleccionado.

Se recurrió a técnicas de medición de variables y a la estadística como ciencia específica en la organización e interpretación de los datos. Para el efecto, se utilizó el Programa Estadístico para las Ciencias Sociales (Statistic Package for Social Sciencies -SPSS-) versión 23. Se utilizó la escala tipo Likert en el instrumento principal para calificar los indicadores de las tres variables. Los enunciados fueron planteados de forma positiva en su totalidad, ante los cuales se solicitó a coordinadores de carreras, el respectivo permiso para su aplicación a estudiantes que albergan. Dicha escala se representó con valores de 1 a 5 , y las respuestas tuvieron dirección favorable o positiva, asignándose dichos valores de la siguiente forma:

C.uadru 1

Escala de Likert para analizar valores

\begin{tabular}{|l|c|}
\hline \multicolumn{2}{|c|}{ Faverable o pesitixa } \\
\hline Nurea & 1 \\
\hline Raru vez & 2 \\
\hline Algubas veces & 3 \\
\hline Casi sientape & 4 \\
\hline Siempre & $\mathbf{5}$ \\
\hline
\end{tabular}

Fuente: Flaboracion propia, oun hase en Blanco y Alvarado \{zovs?.
Los datos se analizaron a través de la estadística descriptiva y se presentaron los resultados a través de tablas de contingencia, gráficos y de manera textual.

Merece la pena señalar que por ser ésta, una investigación mixta, permitió integrar, en un mismo estudio, metodologías cuantitativas y cualitativas, para una mayor comprensión acerca del objeto de estudio.

Por lo que la observación a los grupos investigados y las entrevistas a coordinadores de carreras, que además fungen como docentes de los mismos, fueron necesarias como metodología para obtener datos cualitativos.

El grado de confiabilidad del instrumento, se determinó mediante la aplicación de pruebas estadísticas para obtener el coeficiente Alfa de Cronbach. Se realizó una prueba piloto con la aplicación del instrumento, Inventario SISCO del Estrés Académico a 30 estudiantes, con la intención de conformar base de datos. La escala para interpretar el coeficiente de confiabilidad de la prueba, fue la siguiente:

Cuadró 2

Escala para interpretar el Coeficiente de Confiabilidad

\begin{tabular}{|l|l|}
\hline Rangas & Niveles de Confiabilidad \\
\hline 0.81 a 1.00 & Muy alta \\
\hline 0.61 a 0.80 & Alta \\
\hline 0.41 a 0.60 & Moderada \\
\hline 0.21 a 0.40 & Baja \\
\hline 0.01 a 0.21 & Muy baja \\
\hline
\end{tabular}

Foente: Elaboración propia, con base en Blanioo y Alvarado (2005).

En la presente investigación, los resultados del instrumento presentaron distribución normal y se encontró una confiabilidad con índices alfa de Cronbach de 0.877 para 30 elementos o sujetos de investigación, en relación a 31 ítems que conlleva el cuestionario usado.

En este estudio no se extrajo ninguna muestra de la población, por no ser un grupo muy extenso de estudiantes a investigar; sino que se trabajó con toda la cantidad, 150 alumnos en total, con el objetivo de comprender mejor el contexto, en el cual se relacionan los datos estadísticos; lo que permitió menos errores en su manipulación.

En la presente investigación, los resultados del instrumento presentaron distribución normal y se encontró una confiabilidad con índices alfa de Cronbach de 0.877 para 30 elementos o sujetos de investigación, en relación a 31 ítems que conlleva el cuestionario usado. 
En este estudio no se extrajo ninguna muestra de la población, por no ser un grupo muy extenso de estudiantes a investigar; sino que se trabajó con toda la cantidad, 150 alumnos en total, con el objetivo de comprender mejor el contexto, en el cual se relacionan los datos estadísticos; lo que permitió menos errores en su manipulación.

Atendiendo a lo anterior, se constató lo fructífero de la metodología utilizada a lo largo de la investigación, complemento perfecto para dar respuesta a los objetivos e hipótesis propuestas, lográndose encontrar con veracidad, el perfil del estrés académico, determinado por el uso de estrategias de afrontamiento.

\section{RESULTADOS}

Los resultados derivan del proceso de investigación, sobre el componente "estrategias de afrontamiento", ante el estrés académico que presentan los alumnos de último año de carreras plan fin de semana, del Centro Universitario de Oriente.

Las estrategias de afrontamiento utilizadas, se encuentran según análisis de frecuencias y porcentajes en un nivel "Eventual".

\section{Cuadro.}

Nisel de estratepias de aftrontamienti

\begin{tabular}{|l|c|c|}
\hline Viselinterpretación & Frawencia & Porcentaje válido \\
\hline Esporadico & 13 & $8.7 \%$ \\
\hline Eventual & 71 & $47.3 \%$ \\
\hline Recurrente & 59 & $39.3 \%$ \\
\hline Permanente & 7 & $4.7 \%$ \\
\hline Total & 150 & $100.0 \%$ \\
\hline
\end{tabular}

losute: alabescion propia

De acuerdo con los resultados obtenidos en la variable estrategias de afrontamiento, se permite asegurar que dichas estrategias, sean estas, las enfocadas a la resolución de problemas, o las enfocadas a la regulación emocional.

Son utilizadas por los estudiantes de las carreras investigadas, en un $47.3 \%$ conforme análisis de frecuencias y porcentajes, en un nivel "eventual" según los rangos establecidos.

El cual distribuido entre las carreras tomadas en cuenta en la investigación, quedó de la siguiente manera, según lo indica la tabla posterior.
Cuarin 4

Relacion existente entre la variable estrategias de afrontamiento t tipo de carrera que cursan

\begin{tabular}{|c|c|c|c|c|c|}
\hline \multirow{2}{*}{ Tipo de cartera } & \multicolumn{4}{|c|}{ Fstrategias de Afrostamicnto $\mathrm{H} 3$} & \multirow{2}{*}{ Total } \\
\hline & Esporídica & Excntual & Recurrente & Permaneate & \\
\hline $\begin{array}{l}\text { Licesiatura cn Ciencias de la } \\
\text { Comunicación }\end{array}$ & $\begin{array}{c}2 \\
1.3 \% \\
\end{array}$ & $\begin{array}{c}6 \\
4.0 \% \\
\end{array}$ & $\begin{array}{c}3 \\
2.0 \% \\
\end{array}$ & $\begin{array}{c}1 \\
0.7 \% \\
\end{array}$ & $\begin{array}{c}12 \\
8.0 \% \\
\end{array}$ \\
\hline $\begin{array}{c}\text { Licencialura en Pedagogia y } \\
\text { Administración Educationa }\end{array}$ & $\begin{array}{c}4 \\
2.70 \% \\
\end{array}$ & $\begin{array}{c}18 \\
12.0 \% \\
\end{array}$ & $\begin{array}{c}24 \\
16.0 \% \\
\end{array}$ & $\begin{array}{c}2 \\
1.3 \% \\
\end{array}$ & $\begin{array}{c}48 \\
32.0 \% \\
\end{array}$ \\
\hline $\begin{array}{l}\text { Liecticiatura en } \\
\text { Administración de Empresas }\end{array}$ & $\begin{array}{c}2 \\
1.3 \% \\
\end{array}$ & $\begin{array}{c}16 \\
10.7 \% \\
\end{array}$ & $\begin{array}{c}12 \\
8.0 \%\end{array}$ & $\begin{array}{c}3 \\
2.0 \% \\
\end{array}$ & $\begin{array}{c}33 \\
22.6 \% \\
\end{array}$ \\
\hline $\begin{array}{l}\text { Licenciatura en Concaduria } \\
\text { Pública y Auditoria }\end{array}$ & $\begin{array}{c}5 \\
3,3 \% \\
\end{array}$ & $\begin{array}{c}31 \\
20.7 \% \\
\end{array}$ & $\begin{array}{c}20 \\
13,3 \% \\
\end{array}$ & $\begin{array}{c}1 \\
0.7 \%\end{array}$ & $\begin{array}{c}57 \\
38.0 \% \\
\end{array}$ \\
\hline Total & $\begin{array}{c}13 \\
8.70 \\
\end{array}$ & $\begin{array}{r}71 \\
47.3 \% \\
\end{array}$ & $\begin{array}{c}59 \\
39.3 \% \\
\end{array}$ & $\begin{array}{c}7 \\
4.7 \% \\
\end{array}$ & $\begin{array}{c}150 \\
1000 \%\end{array}$ \\
\hline
\end{tabular}

Predomina en el nivel eventual, un 20.7\% de aplicación de estrategias de afrontamiento, por la carrera de Contaduría Pública, un $12.0 \%$ por Pedagogía, un $10.7 \%$ por la carrera de Administración de Empresas.

La carrera de Ciencias de la Comunicación, con menos porcentaje.

De igual manera existe una proporción muy representativa en los niveles continuos. En añadidura, existe el $39.3 \%$ de utilización de estrategias de afrontamiento en el nivel recurrente; esto significa que los estudiantes las aplican recurrentemente cuando se ven amenazados por las demandas académicas.

El 39.3\% del nivel recurrente se divide según carreras investigadas de esta manera: la carrera de Pedagogía aplica estrategias de afrontamiento en un $16.0 \%$, la carrera de Contaduría Pública en un 13.3\%, Administración de empresas en un $8.0 \%$ y Ciencias de la Comunicación, con menos representatividad.

También se observa, un $4.7 \%$ de utilización de estrategias de afrontamiento, en carreras mencionadas en el nivel permanente, que según rangos establecidos, significa que este grupo aplica estas estrategias de afrontamiento todo el tiempo; mayormente cuando se presentan demandas a las cuales considera peligrosas para su salud. No así el nivel esporádico, donde la aplicación de estrategias de afrontamiento por las carreras en estudio, es muy escaso.

Partiendo de la estadística descriptiva mediante medias y porcentajes obtenidos, se puede comprobar que las estrategias de afrontamiento, más utilizadas por estudiantes, fueron las siguientes: “con habilidad asertiva", esto conduce a pensar, que este grupo de participantes demuestra una conducta equilibrada; se comunican y defienden sus preferencias, ideas o sentimientos, pero respetando, los deseos y opiniones de los demás; expresan sus pensamientos y sentimientos de forma honesta, directa y correcta.

La otra estrategia de afrontamiento utilizada es, "buscando información sobre la situación", se puede especular que estos estudiantes; buscan toda la información posible, sobre la 
realidad con la que están lidiando. Incluyen en su búsqueda leer acerca del problema, hablar con otras personas, o descubrir lo que han hecho otros en su situación para saber lo que pueden esperar. El estar enterados les ayuda a sentirse preparados y con más confianza y además se dan cuenta de que no son las únicas personas que han pasado por esas dificultades.

La tercera estrategia de afrontamiento más utilizada por estudiantes investigados, fue además, "buscando refugio en la religiosidad". Por lo tanto, se puede presumir que este grupo de estudiantes consideran muy importante la religión, la religiosidad y la espiritualidad en sus vidas, debido a que posiblemente en ella, encuentran un mayor contento existencial y una esperanza a la resolución de sus problemas.

\section{DISCUSIÓN}

Se enfatiza en estos apuntes finales, algunos aspectos más relevantes del artículo. Se profundizó en el estudio del fenómeno en estudiantes mencionados, siendo este un grupo que hasta el momento es el menos tomado en cuenta en esta clase de investigaciones, no encontrándose en la búsqueda bibliográfica científica, un sólo trabajo que tome en cuenta a estudiantes de carreras plan fin de semana, a nivel nacional e internacional. Hallándose únicamente un estudio en relación, el cual toma en cuenta el primer año de estudios y el último, pero no con estudiantes del plan fin de semana.

Encontrar el nivel de frecuencia con que se presenta el componente "Estrategias de Afrontamiento" en el estrés académico, de estudiantes investigados, fue uno de los verdaderos aportes de esta investigación, ya que es el componente que determina el nivel de estrés presente en ellos, además muestra cuáles y en qué cantidad fueron utilizadas por los participantes.

Asimismo en referencia a lo anterior, existen otras investigaciones sobre el estrés académico y su componente estrategias de afrontamiento, con resultados similares a esta investigación; en ese sentido, cuando los alumnos están estresados suelen enfrentar su estrés con la "asertividad" (Correa Prieto, 2015), "buscando información sobre la situación" y "buscando refugio en la religiosidad (oraciones o asistencia a misa)" (Nieves, Z., Otero, I., Mabiala, C., Malonda, H. , \& Guimbi, U. 2014).

El estrés académico está presente en alto nivel e intensidad, con un nivel severo $82.7 \%$ según rangos y criterios establecidos, y una intensidad mediamente alta, por lo que los mecanismos para eliminarlo o disminuirlo, no han sido eficaces en estos grupos de estudiantes participantes. La Unión Europea y la Organización Mundial de la Salud, insisten en que se implementen estrategias para la prevención y el control del estrés, en las instituciones. Indicando que muchos son los que padecen estrés y nunca han sido asistidos, por ninguna entidad promotora de salud (Marulanda, 2007).

\section{CONCLUSIONES}

La Constitución Política de la República de Guatemala, en sus artículos, uno, dos, noventa y tres y noventa y cuatro, se refier a la salud física, psíquica y desarrollo integral de toda persona; asimismo indica que es obligación del Estado desarrollar a través del Ministerio de Salud Pública y Asistencia Social, en coordinación con las instituciones estatales, centralizadas, descentralizadas y autónomas, comunidades organizadas y privadas, acciones de promoción, prevención, recuperación de la salud física y mental de sus ciudadanos. Sin dejar de mencionar lo que reza sobre salud mental en el Código de Salud decreto 90-97 y la política de salud mental existente. Muchos de los cambios propuestos para combatir el estrés están al alcance de las manos, basta con el apoyo personal, familiar, profesional o institucional.

Como se versó anteriormente, el plan de estudios, de carreras fin de semana aporta elevados niveles de estrés académico a sus estudiantes. Se evidencia que las estrategias de afrontamiento no fueron efectivas, debido a que el empleo de las mismas no redujo el nivel de estrés autopercibido. Por lo que se considera que no fueron utilizadas en la medida correspondiente, no tienen el suficiente conocimiento sobre ellas, no saben cómo usarlas apropiadamente, o no usan las estrategias adecuadas para cada caso.

\section{REFERENCIAS}

Correa, F.R. (2015). Estrés académico en estudiantes de medicina de la Universidad Cesar Vallejo, de Piura 2013. Revista del Cuerpo Médico del HNAAA, 8(2), 80-84.

Guatemala, Constitución. (1986). Constitución Política de la República de Guatemala. Guatemala: Piedra Santa.

Labrador, F., Crespo, M., Cruzado, J., \& Vallejo, M. (1995). Evaluación y tratamiento de los problemas de estrés. Cádiz: SÍNTESIS.

Lazarus, R. \& Folkman, S. (1986). Estrés y procesos cognitivos. Barcelona: Martínez Roca.

Marulanda, I. (2007). Estrés laboral enemigo silencioso de la salud mental y la satisfacción con la vida. Bogotá: Uniandes.

Nieves, Z., Otero, I., Mabiala, C., Malonda, H., \& Guimbi, U. (2014). Principales manifestaciones del estrés académico en estudiantes de la carrera de medicina de la Universidad "Onze de novembro" Cabinda, Angola. Revista Virtual de Ciencias Sociales y Humanas "PSICOESPACIOS”, 8(12), 402 420.

Ticona, S., Paucar, G., \& Llerena, G. (2010). Nivel de estrés y estrategias de aprontamiento en estudiantes de la facultad de enfermería: UNSA Arequipa. 2006. Enfermería Global, 1-18. 


\section{Sobre autor}

\section{Humberto González Castillo}

Es escritor Chiquimulteco, catedrático de la Escuela Nacional de Maestros de Educación Musical Chiquimula, Docente de la carrera Ciencias de la Comunicación CUNORI-USAC (desde hace 4 años), Licenciado en Pedagogía y Administración Educativa egresado de la Universidad de San Carlos de Guatemala, Maestría en
Docencia Universitaria con Orientación en Estrategias de Aprendizaje, egresado de Estudios de Postgrado CUNORIUSAC.

Copyright (c) 2019 Humberto González Castillo

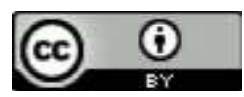

Este texto está protegido por una licencia Creative Commons 4.0.

Usted es libre para Compartir - copiar y redistribuir el material en cualquier medio o formato $-\mathrm{y}$ Adaptar eldocumento - remezclar, transformar y crear a partir del material- para cualquier propósito, incluso comercialmente, siempre que cumpla la condición de:

Atribución: Usted debe reconocer el crédito de una obra de manera adecuada, proporcionar un enlace a la licencia,e indicar si se han realizado cambios. Puede hacerlo en cualquier forma razonable, pero no de forma tal que sugieraque tiene el apoyo del licenciante o lo recibe por el uso que hace.

$\underline{\text { Resumen de licencia }}$ - Texto completo de la licencia 